\title{
EDITORIAL
}

\section{Neuroendocrinology and mental illness}

The interactions between the brain and endocrine system have long been the subject of study. Treatment of psychiatric patients with glandular extracts has been essayed on innumerable occasions, but the hormones "have often been used indiscriminately and without endocrinological investigation of the patient, in the expectation of miraculous results which rarely occurred' (Reiss, 1951). Nevertheless, at that time studies of the relationship between the nervous system and endocrine glands showed great promise in the understanding and treatment of mental illness. Has this promise been justified ? Not entirely, although the reasons underlying the early disappointments are now becoming clear.

As in so many other fields, progress in neuroendocrinology has had to wait upon the development of sensitive and practical methods of hormone assay. And particularly upon the invention of methods that require such small volumes of blood and tissue fluids that changes in hormone secretion can be followed over long periods of time by the collection of serial samples. Newly refined radio immunological assay methods now make it possible to trace the blood level of many hormones in this way, and the information being collected is both fascinating and of great importance.

The lability of hormone secretion is striking. The existence of a circadian variation in the release of pituitary and adrenal hormones has long been known, but when the intervals between sample collection from an individual are shortened it becomes evident that hormones seldom flow from a gland at a particular rate, but tend to be ejected in spurts. Thus assay of the corticosteroid content of plasma collected every 20 minutes over a period of 26 hours has clearly demonstrated the occurrence of oscillations in corticosteroid concentration superimposed upon the familiar circadian (nycthemeral) rhythm. The frequency and amplitude of the oscillations increased during the night. Curiously, similar oscillations persisted in cases of Cushing's syndrome, although the circadian rhythm was lost (Sederberg-Olsen et al., 1973). The realization that hormone secretion is often episodic in character is particularly applicable to the pituitary gland, from which it follows that procedures for assessing the secretion of a trophic hormone may need to be revised. Because of the pulsatile nature of the secretion of luteinizing hormone (LH), for example, the collection of a single sample may coincide with a transiently high plasma concentration, with a nadir in output, or any point between these two extremes. Misleading conclusions may be drawn in this form of lucky dip, so that if only an indication of the level of gonadotrophin secretion is required, measurement of the 24 hour urinary excretion may be more reliable. In a recent study, Santen and Bardin (1973) found that at least three hours of observation, with the collection of blood samples every 20 minutes, was required to determine the mean level of luteinizing hormone in serum with $95 \%$ confidence limits of $\pm 18 \%$. Alternatively, nine consecutive daily samples were needed to provide $95 \%$ confidence limits of less than $\pm 25 \%$. More surges of LH occurred during the follicular phase of the menstrual cycle than during the luteal phase, although the spikes of LH observed during the luteal phase were bigger than those seen earlier in the cycle. Observations of this kind help to account for the wide range of normal values recorded for the blood level of this gonadotrophin, while the continued occurrence of a pulsatile release of $\mathrm{LH}$ in cases of enhanced or depressed release of gonadotrophin needs to be borne in mind. Changes in the pattern of hormone secretion may be of greater significance. Interestingly, Santen and Bardin (1973) recorded secretory spikes of $\mathrm{LH}$, like those normally seen during the luteal phase of the menstrual cycle, in women with psychogenic amenorrhoea, whereas there was relatively little fluctuation in the serum of patients with anorexia nervosa.

As is also demonstrable in animal studies, the hypersecretion of gonadotrophin in agonadal or 
hypogonadal adolescents and adults is also episodic in character (Root et al., 1972), so reflecting the operation of a phasic neural mechanism which is active in the absence of any feedback action of gonadal hormones. Repetitive surges in the secretion of FSH and of LH, with a frequency that varies from individual to individual, also occur in men (Naftolin et al., 1972; Nankin and Troen, 1972). The location and nature of the driving process underlying this pulsatile secretion remains to be determined and presents a most intriguing problem.

The episodic release of anterior pituitary hormones during sleep is particularly marked, yet follicle-stimulating hormone (FSH), LH, growth hormone, and adrenocorticotrophic hormone (ACTH) are not necessarily secreted in unison. In one comparison (Rubin et al., 1973), FSH secretion was not clearly related to the stages of sleep, or to the discharge of $\mathrm{LH}$, which was somewhat higher during REM sleep, whereas the plasma concentration of growth hormone rose with the onset of slow wave sleep early in the sleep period. Adrenocorticotrophic hormone secretion generally increases towards waking. Observations of this kind indicate that separate control mechanisms, and releasing factors, govern the discharge of these trophic hormones and establish that the mechanisms themselves are more complex than anticipated. A further point of interest is that there seems to be an increase in the number of episodes of secretion of LH during the night in pubertal children, so that the mean LH concentration during sleep could be several times that during wakefulness in children early in puberty. This difference is not evident in prepubertal children, or in adults (Boyar et al., 1972).

In view of the lability of pituitary hormone secretion under seemingly resting conditions, it is to be expected that the activity of the hypophysis is affected by many stimuli. This expectation has been realized to the full. At one time stress was equated with the release of ACTH alone, but now it emerges that growth hormone, $\mathrm{LH}$, prolactin, and perhaps thyrotrophic hormone may all be secreted as well. The stressful stimulus need not be especially harmful : a painless dental examination has been found to raise the plasma level of growth hormone markedly. In this context, the effect of emotional deprivation in stunting growth in children, deprivation dwarfism, merits note, for there is evidence of an inhibition of growth hormone secretion in these circumstances, perhaps associated with a disturbance of sleep pattern (Gardner, 1972). Since prolactin secretion is normally inhibited by a hypothalamic factor, the release of this hormone by stressful stimuli implies the removal of a restraining influence, as does the action of a tranquillizer, such as chlorpromazine, in eliciting prolactin discharge.

It should now be less surprising that the common condition of premenstrual tension, which appears to have an endocrine basis, has proved to be a difficult problem to resolve. Attempts to relate the psychological and physical symptoms of premenstrual tension to the rhythmic changes in gonadal hormone secretion have proved unrewarding, particularly as the symptoms do not necessarily subside after ovariectomy or after the menopause (Dalton, 1964). Alternative explanations have been sought, so that on the basis of changes in body weight and in the excretion of sodium and potassium, as well as from evidence in the literature, Janowsky et al. (1973) have argued that quite different interactions, involving the renin-angiotensin-aldosterone system, might underlie this condition. As noted earlier, in connection with the secretion of $\mathrm{LH}$, patterns of brain activity normally associated with a particular pattern of target organ, or gonadal, hormone secretion may be manifest in the absence of that secretion.

In the development and application of highly sensitive radio immunological techniques of hormone assay most effort has naturally been directed toward determining the normal patterns of endocrine gland activity. That phase is coming to an end, but has provided sufficient grounds for the conclusion that neuroendocrinology does indeed have much to offer in the study of mental illness. It remains to turn the promise into performance.

B. T. DONOVAN 


\section{REFERENCES}

Boyar, R., Finkelstein, J., Roffwarg, H., Kapen, S., Weitzman, E., and Hellman, L. (1972). Synchronization of augmented luteinizing hormone secretion with sleep during puberty. New England Journal of Medicine, 287, 582-586.

Dalton, K. (1964). The Premenstrual Syndrome. Heinemann Medical: London.

Gardner, L. I. (1972). Deprivation dwarfism. Scientific American, 227, No. 1, 76-82.

Janowsky, D. S., Berens, S. C., and Davis, J. M. (1973). Correlations between mood, weight, and electrolytes during the menstrual cycle: a renin-angiotensin-aldosterone hypothesis of premenstrual tension. Psychosomatic Medicine, 35, 143-152.

Naftolin, F., Yen, S. C. C., and Tsai, C. C. (1972). Rapid cycling of plasma gonadotrophins in normal men as demonstrated by frequent sampling. Nature, New Biology, 236, 92-93.

Nankin, H. R., and Troen, P. (1972), Overnight patterns of serum luteinizing hormone in normal men. Journal of Clinical Endocrinology and Metabolism, 35, 705-710.

Reiss, M. (1951). Application of endocrinological research methods in psychiatry. Journal of Endocrinology, 7, 235241.

Root, A., DeCherney, A., Russ, D., Duckett, G., Garcia, C.-R., and Wallach, E. (1972). Episodic secretion of luteinizing and follicle-stimulating hormones in agonadal and hypogonadal adolescents and adults. Journal of Clinical Endocrinology and Metabolism, 35, 700-704.

Rubin, R. T., Gouin, P. R., Kales, A., and Odell, W. D. (1973). Luteinizing hormone, follicle stimulating hormone, and growth hormone secretion in normal adult men during sleep and dreaming. Psychosomatic Medicine, 35, 309-321.

Santen, R. J., and Bardin, C. W. (1973). Episodic luteinizing hormone secretion in man. Pulse analysis, clinical interpretation, physiologic mechanisms. Journal of Clinical Investigation, 52, 2617-2628.

Sederberg-Olsen, P., Binder, C., Kehlet, H., Neville, A. M., and Nielsen, I. M. (1973). Episodic variation in plasma corticosteroids in subjects with Cushing's syndrome of differing etiology. Journal of Clinical Endocrinology and Metabolism, 36, 906-910. 